\title{
MiR-22-3p Expression is down-regulated in lung adenocarcinoma
}

\author{
Dong-Jie Ma, Xiao-Yun Zhou, Ying-Zhi Qin, Zhen-Huan Tian, Hong-Sheng Liu \\ and Shan-Qing Liø
}

\author{
Department of Thoracic Surgery, Peking Union Medical College Hospital, CAMS and PUMC, Beijing, 100730, China
}

Background: Our current study was performed with an attempt to detect the expression of microRNA-22-3p (miR-22-3p) in lung adenocarcinoma, as well as to analyze its role in clinical practice. In addition, its relationship with vascular endothelial growth factor (VEGF) and metastasis related indexes was focused. Material and Method: The trials in which 62 cases of lung adenocarcinoma were received to collect tumor tissue (study group) and normal lung tissue (control group) were eligible for this study. The expression of miR-22-3p in the two groups was detected through RT-PCR. Immunohistochemical method was used to detect the expression of VEGF and leukocyte differentiation antigen 31 (CD31) marked microvessel density (MVD) in lung adenocarcinoma. The expressions of matrix metalloproteinase-3 (MMP-3) and matrix metalloproteinase-7 (MMP-7) in lung adenocarcinoma were also detected through the use of Western Blot. Results: The present study revealed significant difference in the expression of miR-22-3p between the two groups. No significant difference in the expression of gender, age, neural invasion and the number of lesions were observed between groups. There was significant difference in the expression of miR-22-3p in the maximum diameter of tumor, pleural recidivism, vascular recidivism, lymph node metastasis and different TNM stages. Based on survival analysis, miR-22-3p was linked to survival time. Correlation analysis indicated that there was negative correlation between miR-22-3p and VEGF, miR-22-3p and MVD, miR-22-3p and MMP-3, and miR22-3p and MMP-7 in lung adenocarcinoma. Conclusion: Our findings provide evidence that miR-22-3p is low expressed in lung adenocarcinoma tissues and the low expression of miR-22-3p is closely associated with clinicopathological characteristics and the prognosis. MiR22-3p may be involved in the tumor progression of lung adenocarcinoma and may serve as a biomarker for the diagnosis and prognosis of lung adenocarcinoma.

Key words: lung adenocarcinoma, prognosis of miR-22-3p, VEGF, MMPs

Received: 06 November, 2020; revised: 17 December, 2020; accepted: 18 December, 2020; available on-line: 08 July, 2021

『e-mail: Penny6527@sohu.com

Abbreviations: miRNAs, Micro-RNA, MVD, marked microvessel density; VEGF, vascular endothelial growth factor

\section{INTRODUCTION}

The occurrence of lung adenocarcinoma is considered to be closely associated with the imbalance of gene and protein expression, which involves a variety of molecular biological mechanisms, such as DNA, RNA and so on
(Yang et al., 2016). Micro-RNA (miRNAs) is a non-coding single-stranded small molecule RNA with a length of 21-25 nucleotides, which is related to the formation of epithelial malignant tumors based on several studies in recent years. It binds to the 3'UTR coding region of the target mRNA sequence, leading to the regulation of downstream factors after mRNA degradation to play a biological role (Cho et al., 2009; Sakamoto et al., 2014). TCGA database was utilized to screen the expression of miRNAs in lung adenocarcinoma (Zhao et al., 2019). The small RNA-22-3p (miR-22-3p) was selected in our study for its potential involvement in lung adenocarcinoma, which has not yet been reported. Meanwhile, miRtarbase was used to predict target factors, and the most correlated upper vascular endothelial growth factor (VEGF), CD31 marked microvessel density (MVD), matrix metalloproteinase-3 (MMP-3) and matrix metalloproteinase-7 (MMP-7) were selected for this experiment. The current study seeks to explore miR-22-3p and its regulatory effect on angiogenesis and metastasis by conducting a prospective experimental study as a target to identify its potential role in clinical practice.

\section{METHODS AND MATERIALS}

\section{Participants}

Totally, there were 62 patients diagnosed with lung adenocarcinoma in our hospital from November, 2013 to August, 2014 as subjects. To be included in the current study, the following inclusion criteria should be met: 1) patients were diagnosed with lung adenocarcinoma through postoperative pathology, in accordance with the diagnostic criteria of WHO; 2) patients were firstly diagnosed and had receiving surgical intervention; 3) complete clinical data. Exclusion criteria were as follows: 1) unclear diagnosis; 2) preoperative treatments for tumor; 3) metastatic adenocarcinomas; 4) palliative operations. There were 33 males and 29 females in total, aged 33 to 80 years, with a median age of 61 years. Among them, 29 cases had pleural invasion and 33 cases had no pleural invasion. There were 20 cases with lymph node metastasis and 42 cases without lymph node metastasis. The tumor tissue was used as the study group and the normal lung tissue larger than $3 \mathrm{~cm}$ away from tumor edge as the control group. The fresh specimens were frozen in the refrigerator at $-80^{\circ} \mathrm{C}$ and the paraffin-embedded tissues were retained. The study was approved by the Hospital Ethics Committee and the families of patients signed informed consent forms. 


\section{Evaluation of sample size}

The sample inclusion formula of quantitative data was applied: $\mathrm{n}_{1}=\mathrm{n}_{2}=2\left[\left(\mathrm{u}_{\alpha}+\mathrm{u}_{\beta}\right) / \delta / \sigma\right]^{2}+1 / 4 \mathrm{u}_{\alpha}{ }^{2}$. Relevant literatures were screened for the pre-experimental results. Our study predicted that the expression level of tumor tissue and normal tissue was 1.2 and 1.8 respectively, and $\sigma=0.6, \delta / \sigma=0.5, \alpha$ was 0.05 as median, and $\mathrm{n}_{1}=\mathrm{n}_{2} \approx 62$ cases.

\section{Detection of MiR-22-3p}

Fresh samples were detected by real-time fluorescence quantitative PCR (real-time fluorescence quantitative PCR, qPCR). The primers were synthesized by Shanghai Laifeng Biotechnology Co., Ltd., with upstream: 5'-TGACAACCGT'T'T'TGACTG-3' and downstream: 5'-TACTGT'T'TGAAAATCGT'T-3'. U6 small nuclear RNA was used as internal reference, with upstream: 5'-GGAACAGAGAAGATTA GC-3', and downstream: 5'-T'TGGAATCACGAAT'TCCG-3'. The total RNA was extracted from fresh tissue by TRIzol to measure the concentration of RNA and the integrity of RNA. $1 \mu \mathrm{g}$ of total RNA was used to generate cDNA strand. CDNAs was synthesized from TaqMan MicroRNA reverse transcription kit. The reagent was purchased from Shanghai Laifeng Biotechnology Co., Ltd. and ABI Company of the United States. The conditions for reverse transcription were $40^{\circ} \mathrm{C}$ for $15 \mathrm{~min}$ and $90^{\circ} \mathrm{C}$ for $5 \mathrm{sec}$. Program setting was as follows: $95^{\circ} \mathrm{C}$ for 10 minutes for one cycle, $95^{\circ} \mathrm{C}$ for 30 seconds, $59^{\circ} \mathrm{C}$ for 30 seconds, and $72^{\circ} \mathrm{C}$ for 30 seconds. The abovementioned steps experienced a total of 35 cycles, with $72^{\circ} \mathrm{C}$ for 7 min for extension. The fluorescence signal was collected at $60^{\circ} \mathrm{C}$ and the CT value was recorded. The results were expressed as $2^{-\Delta \Delta \mathrm{Ct}}=[\mathrm{Ct}$ target gene (unknown sample)-Ct GAPDH (unknown sample)]-[Ct target gene (calibration sample)Ct GAPDH (calibration sample)].

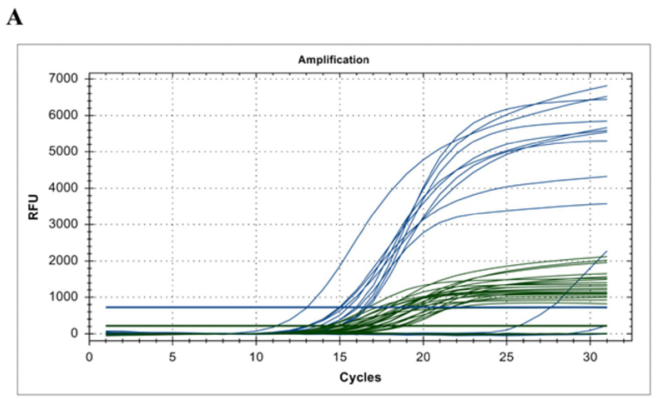

B

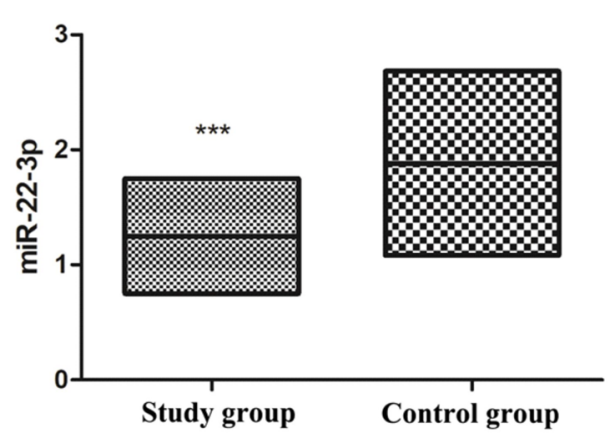

\section{Detection of VEGF and CD31}

Immunohistochemical two-step method was used to detect the expression of VEGF and CD31 in lung adenocarcinoma. Immunohistochemical analysis of VEGF and CD31 was detected in paraffin embedded $4 \mu \mathrm{m}$ sections of lung adenocarcinoma using $15 \mu \mathrm{g} / \mathrm{mL}$ VEGF or CD31 Antibody overnight at $4^{\circ} \mathrm{C}$. Tissue was stained with the Anti-Goat HRP-DAB (brown and counterstained with hematoxylin (blue). VEGF, CD31 antibody and DAB chromogenic agents were purchased from Thermo Fisher Scientific and Beijing Zhongshan Jinqiao Biological Co., Ltd. Every experimental procedure was strictly conducted. Results was interpreted by pathologist and blind method was used. The expression site of VEGF was cytoplasm, with brownish yellow as positive. Five hot spots were counted (400-fold), and the average value was taken as the positive rate. The expression site of CD31 was the cytoplasm and/or cell membrane of vascular endothelial cells, which was determined as microvessels by continuous cell clusters, cell plexus or branched endothelial cells. The microvessel density (MVD) was counted in 5 tumor stromal regions (400-fold), and the average value was taken as the result of MVD. The number of positive-staining cells in ten representative microscopic fields was counted and the percentage of positive cells was calculated. Given the homogenicity of the staining of the target proteins, tumor specimens were scored in a semiquantitative manner based on the percentage of tumor cells that showed immunoreactivity.

\section{Detection of MMP-3 and MMP-7}

Western Blot method was utilized to detect the expression of MMP-3 and MMP-7 in lung adenocarcinoma. Fresh frozen samples were used for protein extraction, concentration determination, denaturation, addition, electrophoresis, electroporation and immune hybridization

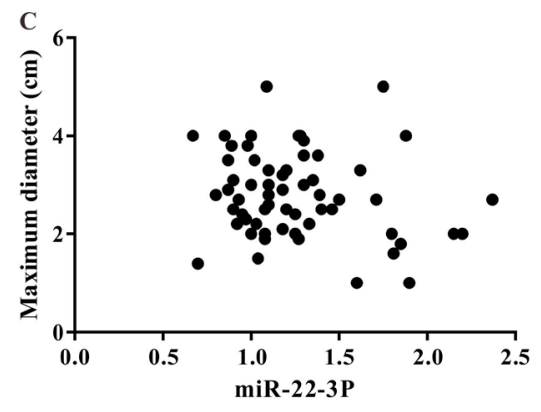

D

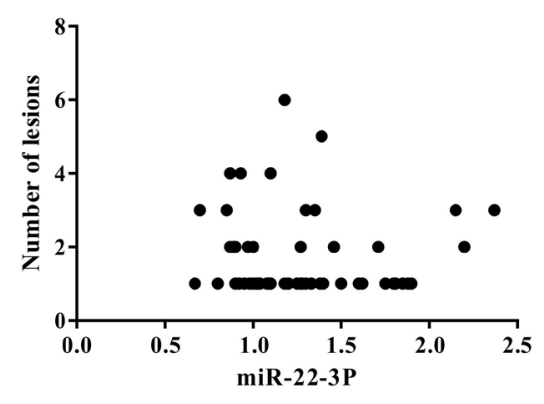

Figure 1. Expression of miR-22-3p in the control and study group (qPCR method).

(A) qPCR results. (B) Expression of miR-22-3p in the control and study group. (C) Scatter plots of miR-22-3p and maximum diameter of lesions. (D) Scatter plot of miR-22-3p and number of lesions. ${ }^{* * *} P<0.05$ compared with the control group. 
according to experimental process. $30 \mu \mathrm{g}$ of total protein was used. The MMP-3, MMP-7 and GAPDH primary antibody and HRP-conjugated secondary antibody. were purchased from Abcam, UK. All antibodies were dissolved in antibody dilutions (Beyotime, Shanghai, China, P0023A) at a ratio of 1:1000. After taking out the film and adding the luminous solution, the film was exposed for 2 minutes and developed for 20 seconds. The ratio of target protein to GAPDH was analyzed by Image J software.

\section{Statistical analysis}

SPSS13.0 was used for analysis, $t$ test, paired $t$ test and analysis of variance (SNK method for pair comparison). Pearson correlation analysis and Kaplan-Meier survival analysis were applied and a $P<0.05$ represented significant difference.

\section{RESULTS}

\section{Comparison of miR-22-3p expression between the two groups}

There was significant difference in the expression of miR-22-3p between the two groups $(P<0.05)$. The expression of miR-22-3p in the study group was significantly lower than that in the control group. See Fig. 1.

\section{Comparison of miR-22-3p expression in different clinicopathological features}

Significantly differences of the expression of miR-22$3 \mathrm{p}$ were observed in tumor maximum diameter, pleural invasion, vascular invasion, lymph node metastasis (see Fig. 2) and different TNM stages $(P<0.05)$. There was no significant difference in the expression of gender, age, neural invasion and the number of lesions $(P>0.05)$. See Table 1.

\section{Relationship between miR-22-3p and survival time}

All patients were followed up for 60 months. Updated to June 30, 2019, the survival time was 5 to 60 months, and the median survival time was 22 months. Among them, 15 cases survived, 44 cases died and 3 cases lost follow-up. Survival analysis showed that the expression of $\mathrm{miR}-22-3 \mathrm{p}$ was related to survival time $\left(\mathrm{X}^{2}=5.67\right.$, $P=0.011)$. See Fig. 3.

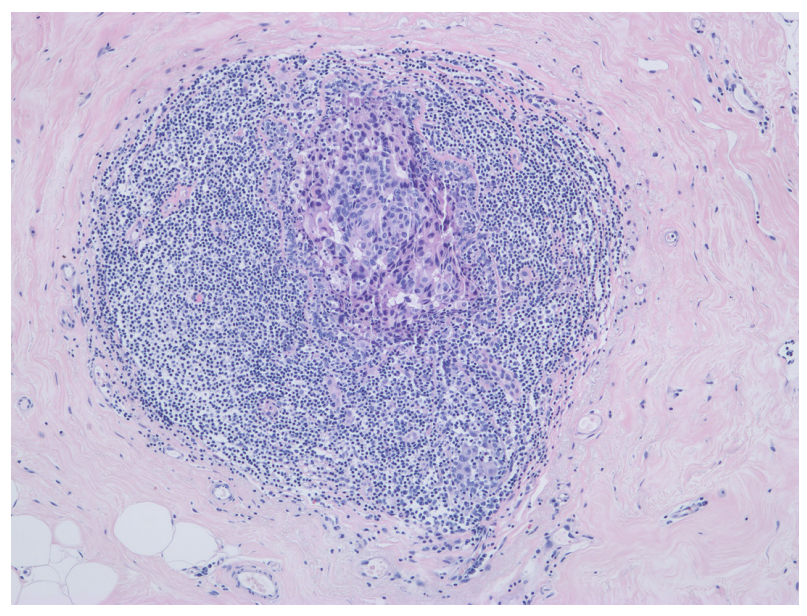

Figure 2. Lymph node metastasis of tumor (HE staining, 200fold magnification).
Table 1. Comparison of miR-22-3p expression in different clinicopathological features

\begin{tabular}{|c|c|c|c|c|}
\hline Clinical features & cases & miR-22-3p & $\mathrm{t}$ & $P$ \\
\hline \multicolumn{3}{|l|}{ Gender } & \multirow{3}{*}{0.98} & \multirow{3}{*}{0.528} \\
\hline male & 33 & $1.28 \pm 0.39$ & & \\
\hline female & 29 & $1.22 \pm 0.35$ & & \\
\hline \multicolumn{3}{|l|}{ Age } & \multirow{3}{*}{0.97} & \multirow{3}{*}{0.532} \\
\hline$<60$ years & 25 & $1.28 \pm 0.38$ & & \\
\hline$\geq 60$ years & 37 & $1.23 \pm 0.31$ & & \\
\hline \multicolumn{2}{|c|}{ Number of lesions } & & \multirow{3}{*}{0.84} & \multirow{3}{*}{0.622} \\
\hline 1 & 42 & $1.24 \pm 0.34$ & & \\
\hline$\geq 2$ & 20 & $1.27 \pm 0.30$ & & \\
\hline \multicolumn{3}{|l|}{$\begin{array}{l}\text { Neural } \\
\text { invasion }\end{array}$} & \multirow{3}{*}{0.96} & \multirow{3}{*}{0.530} \\
\hline$N$ & 52 & $1.24 \pm 0.41$ & & \\
\hline Y & 10 & $1.30 \pm 0.38$ & & \\
\hline Pleural invasion & & 77.5 & \multirow{3}{*}{5.36} & \multirow{3}{*}{0.010} \\
\hline N & 33 & $1.40 \pm 0.31$ & & \\
\hline Y & 29 & $1.07 \pm 0.37$ & & \\
\hline \multicolumn{3}{|l|}{ Vascular invasion } & \multirow{3}{*}{5.02} & \multirow{3}{*}{0.019} \\
\hline N & 51 & $1.30 \pm 0.36$ & & \\
\hline Y & 11 & $1.01 \pm 0.35$ & & \\
\hline \multicolumn{3}{|c|}{$\begin{array}{l}\text { Lymph node meta- } \\
\text { stasis }\end{array}$} & \multirow{3}{*}{7.30} & \multirow{3}{*}{0.003} \\
\hline N & 42 & $1.38 \pm 0.43$ & & \\
\hline Y & 20 & $0.98 \pm 0.42$ & & \\
\hline \multicolumn{3}{|c|}{ Maximum diameter } & \multirow{3}{*}{7.39} & \multirow{3}{*}{0.002} \\
\hline$<3 \mathrm{~cm}$ & 37 & $1.38 \pm 0.44$ & & \\
\hline$\geq 3 \mathrm{~cm}$ & 25 & $0.99 \pm 0.45$ & & \\
\hline \multicolumn{3}{|l|}{ TNM stage } & \multirow{3}{*}{5.30} & \multirow{3}{*}{0.012} \\
\hline$|-I|$ & 32 & $1.41 \pm 0.35$ & & \\
\hline III-IV & 30 & $1.08 \pm 0.39$ & & \\
\hline
\end{tabular}

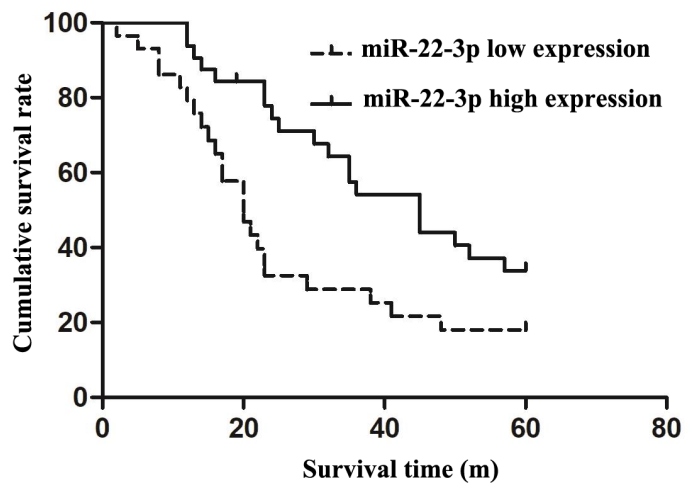

Figure 3. Relationship between miR-22-3p and survival time

\section{Correlation between miR-22-3p and VEGF, and miR-22- $3 p$ and MVD}

The positive rate of VEGF in lung adenocarcinoma ranged from $10 \%$ to $60 \%$, with an average of $33.4 \%$. Correlation analysis showed that there was a negative correlation between the positive rate of miR-22-3p and VEGF in the study group $(r=-0.56, P=0.015)$. The range 

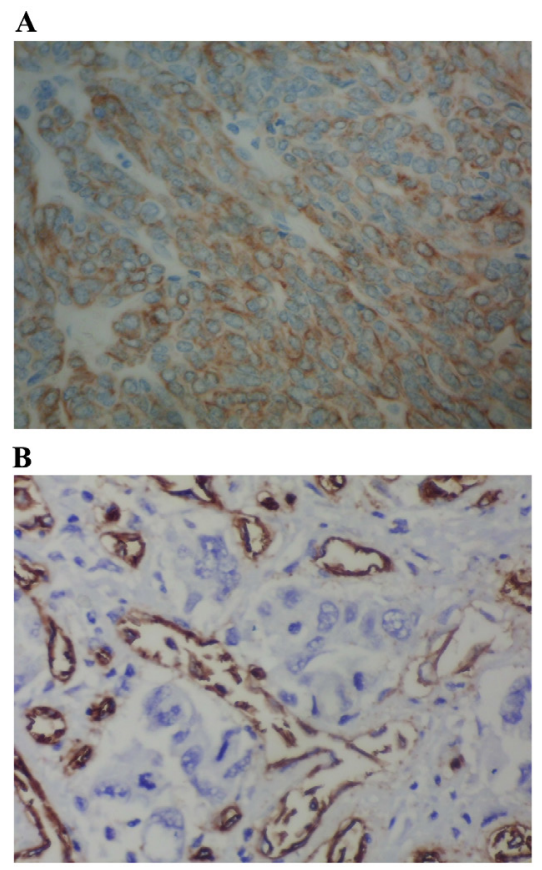

Figure 4. Immunohistochemical diagram.

(A) the expression of VEGF (200-fold); (B) CD31 labeled MVD in tumor stroma (200-fold)

of MVD was 13 to 40, with an average of 22.5. Based on correlation analysis, there was a negative correlation between miR-22-3p and MVD in the study group $(\mathrm{r}=-0.54, P=0.013)$. See Figs $4-5$.

Correlation between miR-22-3p and MMP-3, and miR22-3p and MMP-7

The range of MMP-3 detected by Western Blot was 0.6 to 3.1, with an average of 1.8. Correlation analysis showed that there was a negative correlation between miR-22-3p and MMP-3 ( $r=-0.55, P<0.012)$. The range of MMP-7 was 0.7 to 2.8 , with an average of 1.8 . Correlation analysis revealed that there was a negative correlation between miR-22-3p and MMP-7 ( $\mathrm{r}=-0.50$, $P<0.009)$. See Fig. 6.

\section{DISCUSSION}

Lung adenocarcinoma is a tumor caused by the abnormal regulation following genetic changes. According to recent studies (Flamini et al., 2017; Liu et al., 2017), miRNAs have been proved to play a crucial part in the
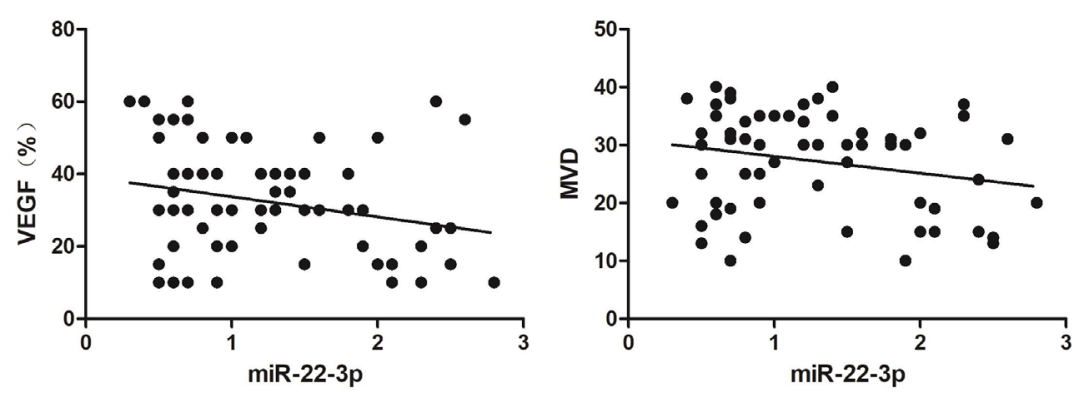

Table 2. Comparison of miR-22-3p expression in different clinicopathological features

\begin{tabular}{|c|c|c|c|c|}
\hline Clinical features & cases & miR-22-3p & $\mathrm{t}$ & $\mathrm{P}$ \\
\hline \multicolumn{3}{|l|}{ Gender } & \multirow{3}{*}{0.98} & \multirow{3}{*}{0.528} \\
\hline male & 33 & $1.28 \pm 0.39$ & & \\
\hline female & 29 & $1.22 \pm 0.35$ & & \\
\hline \multicolumn{3}{|l|}{ Age } & \multirow{3}{*}{0.97} & \multirow{3}{*}{0.532} \\
\hline$<60$ years & 25 & $1.28 \pm 0.38$ & & \\
\hline$\geq 60$ years & 37 & $1.23 \pm 0.31$ & & \\
\hline \multicolumn{3}{|l|}{ Number of lesions } & \multirow{3}{*}{0.84} & \multirow{3}{*}{0.622} \\
\hline 1 & 42 & $1.24 \pm 0.34$ & & \\
\hline$\geq 2$ & 20 & $1.27 \pm 0.30$ & & \\
\hline \multicolumn{3}{|l|}{$\begin{array}{l}\text { Neural } \\
\text { invasion }\end{array}$} & \multirow{3}{*}{0.96} & \multirow{3}{*}{0.530} \\
\hline $\mathrm{N}$ & 52 & $1.24 \pm 0.41$ & & \\
\hline $\mathrm{Y}$ & 10 & $1.30 \pm 0.38$ & & \\
\hline \multicolumn{2}{|l|}{ Pleural invasion } & 77.5 & \multirow{3}{*}{5.36} & \multirow{3}{*}{0.010} \\
\hline $\mathrm{N}$ & 33 & $1.40 \pm 0.31$ & & \\
\hline $\mathrm{Y}$ & 29 & $1.07 \pm 0.37$ & & \\
\hline \multicolumn{3}{|l|}{ Vascular invasion } & \multirow{3}{*}{5.02} & \multirow{3}{*}{0.019} \\
\hline $\mathrm{N}$ & 51 & $1.30 \pm 0.36$ & & \\
\hline $\mathrm{Y}$ & 11 & $1.01 \pm 0.35$ & & \\
\hline \multicolumn{3}{|c|}{$\begin{array}{l}\text { Lymph node meta- } \\
\text { stasis }\end{array}$} & \multirow{3}{*}{7.30} & \multirow{3}{*}{0.003} \\
\hline $\mathrm{N}$ & 42 & $1.38 \pm 0.43$ & & \\
\hline $\mathrm{Y}$ & 20 & $0.98 \pm 0.42$ & & \\
\hline \multicolumn{3}{|c|}{ Maximum diameter } & \multirow{3}{*}{7.39} & \multirow{3}{*}{0.002} \\
\hline$<3 \mathrm{~cm}$ & 37 & $1.38 \pm 0.44$ & & \\
\hline$\geq 3 \mathrm{~cm}$ & 25 & $0.99 \pm 0.45$ & & \\
\hline \multicolumn{3}{|l|}{ TNM stage } & \multirow{3}{*}{5.30} & \multirow{3}{*}{0.012} \\
\hline $\mathrm{I}-1 \mathrm{I}$ & 32 & $1.41 \pm 0.35$ & & \\
\hline III-IV & 30 & $1.08 \pm 0.39$ & & \\
\hline
\end{tabular}

evolution of lung adenocarcinoma, which mainly regulate the downstream target genes with the regulatory function at the transcriptional level. There are several miRNAs with complex mechanism, which not only regulate cell cycle, differentiation, cell proliferation and apoptosis, but also have potential role in metastasis and epithelialstromal transformation. The current study specifically focused on the significance of miR-22-3p expression,

Figure 5. Correlation analysis diagram 

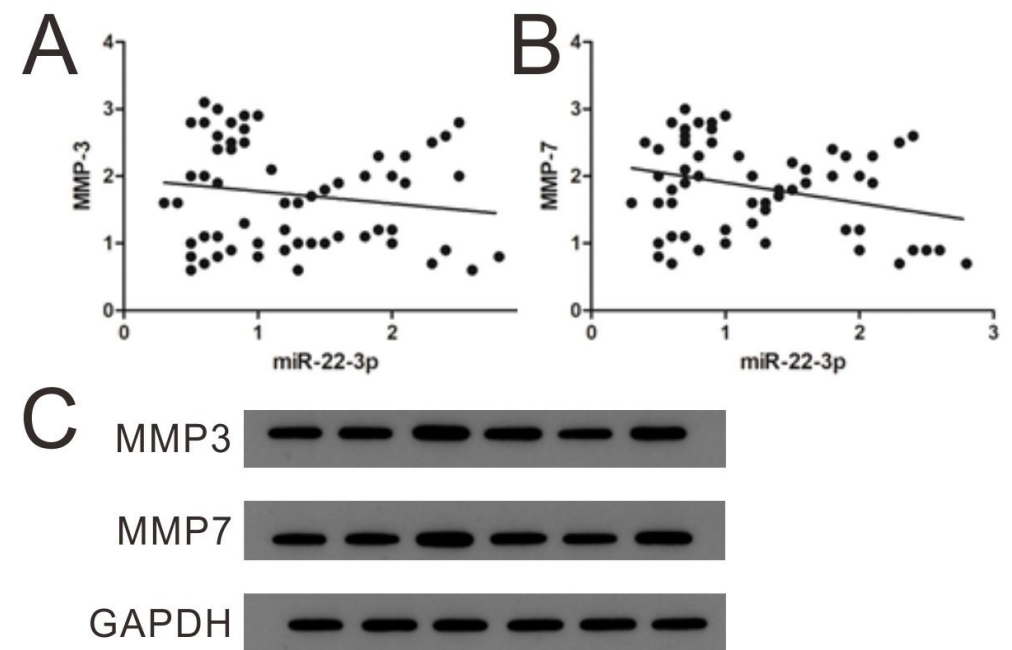

Figure 6. Correlation between miR-22-3p and MMP-3, and miR-22-3p and MMP-7.

(A) Correlation between miR-22-3p and MMP-3. (B) Correlation between miR-22-3p and MMP-7. (C) Western Blot results of MMP-3 and MMP-7 in normal lung tissue.

with an attempt to explore its role in vascular endothelial growth factor and metastasis. VEGF is a factor related to hypoxia, which is up-regulated in malignant tumors. In addition, it mainly interacts with receptor VEGFR2 to induce proliferation and reproduction of vascular endothelial cells, promote interstitial angiogenesis, and provide nutrition and oxygen for tumor growth (Frezzetti et al., 2017; Lin et al., 2017). In this study, CD31 was selected as a marker of vascular endothelial cells, which can objectively and accurately reflect the morphology of angiogenesis and vascular growth. MMP-3 and MMP-7, the classical members of MMPs family, were selected, which increase their expression during tumor growth with the strongest degradation effect on basement membrane and type IV collagen, resulting in obvious destruction of cell metastasis barrier to provide a direct pathway for metastasis (Kim et al., 2005; Zheng et al., 2013).

Based on the results, the low expression of miR-22-3p in lung adenocarcinoma was related to the maximum diameter of the tumor, pleural invasion, vascular invasion, lymph node metastasis and different TNM stages, suggesting that the low expression of miR-22-3p can promote the formation and progress of the tumor, which is similar to the function of tumor suppressor genes. The expression of miR-22-3p could be detected in the epithelial tissues of normal organs, and the expression of miR$22-3 p$ decreases during tumorigenesis, especially obvious with continuous effect in the process of tumor growth, pleural invasion and lymph node metastasis. It was found that the abnormal expression of miR-22-3p was related to VEGF and interstitial MVD, indicating that the low expression of miR-22-3p can increase the production of VEGF. To our knowledge, VEGF acts by binding to the receptor VEGFR2 on its endothelial cell membrane. And VEGFR2 is a transmembrane receptor of endothelial cells, which is a ligand-mediated dimer and can promote the phosphorylation of adjacent receptor subunits, activate signal transduction, and specifically promote the proliferation of endothelial cells. The increase of $\mathrm{Ca}^{2+}$ concentration in endothelial cells by VEGFR2 stimulates the metabolism of inositol phosphate and causes endothelial cells to migrate into collagen and form neovascularization (Sun et al., 2017). In this study, the CD31 neovascularization are single endothelial cells with branch buds and strips, all of which are new endothelial cells that form functional lumen due to physical factors such as blood flow shock. The correlation between miR22-3p and MMP-3, MMP-7 was observed, suggesting that $\mathrm{miR}-22-3 \mathrm{p}$ can regulate metastasis, and its regulation of MMPs is one of the mechanisms. MMP-3 and MMP-7 are the most associated factors with miR-22-3p and the most important downstream genes of miR-22-3p (Hu et al., 2019; Lv et al., 2018). MMP-7 has the strongest effect on the degradation of extracellular matrix, and when MMP-7 is activated, which can regulate interstitial angiogenesis, epithelial-mesenchymal transformation and cell proliferation. Based on this study, no relationship between miR-22-3p and neural invasion was found, possibly due to the weak effect of miR-22-3p on the invasion and interference of nerve fibers (Dai et al., 2019). Moreover, no correlation between miR-22-3p and the number of lung tumor lesions was found, indicating that the abnormal expression of miR-22-3p may not be involved in the intrapulmonary dissemination of the tumor. Given that miR-22-3p is related to survival time, possibility exists that the low expression of miR-22-3p may have potential in predicting the poor prognosis of tumor. Nevertheless, whether miR-22-3p can be used as an independent prognostic factor needs to be further confirmed by more clinical studies. Although studies of wider population are still needed, our study believes that the abnormal expression of miR-22-3p is an important molecular event in the formation of lung adenocarcinoma, with many downstream regulatory factors and regulatory functions (Li et al., 2017; Wang et al., 2018). We recommend an appropriate experimental design based on the awareness of the complexity of miR-22-3p if our findings could be confirmed in a larger follow-up studies to reveal the mechanism of miR-22-3p in lung adenocarcinoma.

Altogether, miR-22-3p is low expressed in lung adenocarcinoma tissues and the low expression of miR-22-3p is closely associated with clinicopathological characteristics and the prognosis. In addition, the expression level of miR-22-3p is related to the degradation of VEGF and extracellular matrix in lung adenocarcinoma. MiR-22-3p may be involved in the tumor progression of lung adenocarcinoma and may serve as a biomarker for the diagnosis and prognosis of lung adenocarcinoma. 


\section{REFERENCES}

Cho WC, Chow AS, Au JS (2009) Restoration of tumour suppressor hsa-miR-145 inhibits cancer cell growth in lung adenocarcinoma patients with epidermal growth factor receptor mutation. Eur I Cancer 45: 2197-2206. https://doi.org/10.1016/j.ejca.2009.04.039

Dai Z, Yan H, Wang K, Zhong S, Zhou M, Zheng D, Zhu H (2019) MicroRNA-22 regulates thyroid cell growth and lipid accumulation via IL6R. Front Biosci (Landmark Ed) 24: 1350-1362

Flamini V, Jiang WG, Cui Y (2017) Therapeutic role of MiR-140-5p for the treatment of non-small cell lung cancer. Anticancer Res 37: 4319-4327. https://doi.org/10.21873/anticanres.11825

Frezzetti D, Gallo M, Maiello MR, D'Alessio A, Esposito C, Chicchinelli N, Normanno N, De Luca A (2017) VEGF as a potential target in lung cancer. Expert Opin Ther Targets 21: 959-966. https://doi. org/10.1080/14728222.2017.1371137

Hu Z, Lv X, Chen L, Gu X, Qian H, Fransisca S, Zhang Z, Liu Q, Xie P (2019) Protective effects of microRNA-22-3p against retinal pigment epithelial inflammatory damage by targeting NLRP3 inflammasome. J Cell Physiol 234: 18849-18857. https://doi.org/10.1002/ jcp. 28523

Kim SJ, Rabbani ZN, Dewhirst MW, Vujaskovic Z, Vollmer RT, Schreiber EG, Oosterwijk E, Kelley MJ (2005) Expression of HIF1alpha CA IX VEGF and MMP-9 in surgically resected non-small cell lung cancer. Lung Cancer 49: 325-335. https://doi.org/10.1016/j. lungcan.2005.03.036

Li C, Ni J, Liu YX, Wang H, Liang ZQ, Wang X (2017) Response of MiRNA-22-3p and MiRNA-149-5p to folate deficiency and the differential regulation of MTHFR Expression in normal and cancerous human hepatocytes. PLoS One 12: e0168049. https://doi. org/10.1371/journal.pone.0168049

Lin CY, Cho CF, Bai ST, Liu JP, Kuo TT, Wang LJ, Lin YS, Lin CC, Lai LC, Lu TP, Hsieh CY, Chu CN, Cheng DC, Sher YP (2017) ADAM9 promotes lung cancer progression through vascular remodeling by VEGFA ANGPT2 and PLAT. Sci Rep 7: 15108. https://doi.org/10.1038/s41598-017-15159-1
Liu L, Bi N, Wu L, Ding X, Men Y, Zhou W, Li L, Zhang W, Shi S, Song Y, Wang L (2017) MicroRNA-29c functions as a tumor suppressor by targeting VEGFA in lung adenocarcinoma. Mol Cancer 16: 50. https://doi.org/10.1186/s12943-017-0620-0

Lv KT, Liu Z, Feng J, Zhao W, Hao T, Ding WY, Chu JP, Gao LJ (2018) MiR-22-3p Regulates cell proliferation and inhibits cell apoptosis through targeting the eIF4EBP3 gene in human cervical squamous carcinoma cells. Int J Med Sci 15: 142-152. https://doi. org/10.7150/ijms. 21645

Sakamoto N, Naito Y, Oue N, Sentani K, Uraoka N, Oo HZ, Yanagihara K, Aoyagi K, Sasaki H, Yasui W (2014) MicroRNA-148a is downregulated in gastric cancer targets MMP7 and indicates tumor invasiveness and poor prognosis. Cancer Sci 105: 236-243. https:// doi.org/10.1111/cas.12330

Sun X, Ma X, Wang J, Zhao Y, Wang Y, Bihl JC, Chen Y, Jiang C (2017) Glioma stem cells-derived exosomes promote the angiogenic ability of endothelial cells through miR-21/VEGF signal. Oncotarget 8: 36137-36148. https://doi.org/10.18632/oncotarget.16661

Wang M, Chen B, Ru Z, Cong L (2018) CircRNA circ-ITCH suppresses papillary thyroid cancer progression through miR-22-3p/CBL/ beta-catenin pathway. Biochem Biophys Res Commun 504: 283-288. https://doi.org/10.1016/j.bbrc.2018.08.175

Yang Y, Xu W, Liu D, Ding X, Su B, Sun Y, Gao W (2016) PTEN polymorphisms contribute to clinical outcomes of advanced lung adenocarcinoma patients treated with platinum-based chemotherapy. Tumour Biol 37: 7785-7796. https://doi.org/10.1007/s13277-0154651-0

Zhao L, Wang Y, Liu Q (2019) Catalpol inhibits cell proliferation invasion and migration through regulating miR-22-3p/MTA3 signalling in hepatocellular carcinoma. Exp Mol Pathol 109: 51-60. https://doi. org/10.1016/j.yexmp.2019.104265

Zheng X, Chopp M, Lu Y, Buller B, Jiang F (2013) MiR-15b and miR152 reduce glioma cell invasion and angiogenesis via NRP-2 and MMP-3. Cancer Lett 329: 146-154. https://doi.org/10.1016/j.canlet.2012.10.026 